\title{
ARTICLE
}

\section{Nanoporous frameworks exhibiting multiple stimuli responsiveness}

\author{
Pintu K. Kundu ${ }^{1}$ Gregory L. Olsen², Vladimir Kiss ${ }^{3} \&$ Rafal Klajn ${ }^{1}$
}

Nanoporous frameworks are polymeric materials built from rigid molecules, which give rise to their nanoporous structures with applications in gas sorption and storage, catalysis and others. Conceptually new applications could emerge, should these beneficial properties be manipulated by external stimuli in a reversible manner. One approach to render nanoporous frameworks responsive to external signals would be to immobilize molecular switches within their nanopores. Although the majority of molecular switches require conformational freedom to isomerize, and switching in the solid state is prohibited, the nanopores may provide enough room for the switches to efficiently isomerize. Here we describe two families of nanoporous materials incorporating the spiropyran molecular switch. These materials exhibit a variety of interesting properties, including reversible photochromism and acidochromism under solventfree conditions, light-controlled capture and release of metal ions, as well reversible chromism induced by solvation/desolvation.

\footnotetext{
${ }^{1}$ Department of Organic Chemistry, Weizmann Institute of Science, 76100 Rehovot, Israel. ${ }^{2}$ Department of Chemical Physics, Weizmann Institute of Science, 76100 Rehovot, Israel. ${ }^{3}$ Department of Biological Chemistry, Weizmann Institute of Science, 76100 Rehovot, Israel. Correspondence and requests for materials should be addressed to R.K. (email: rafal.klajn@weizmann.ac.il).
} 
S timuli-responsive molecules ${ }^{1}$ or nanoparticles ${ }^{2}$ are capable of changing their structure and/or properties in response to external stimuli such as small molecules, light or magnetic field. These entities have recently attracted much attention as key components of stimuli-responsive materials, examples of which include self-darkening windows ${ }^{3}$, self-healing coatings ${ }^{4-6}$, or silica-based delivery vesicles, which can be engineered to release cargo molecules upon activation with a variety of external stimuli $^{7,8}$. Among these different stimuli, light is arguably the most attractive: it is a stimulus that can be delivered instantly, into a precise location, and in the form of different wavelengths, to which different photoresponsive molecules selectively respond $^{9-11}$. One of the most unique examples of molecular switches is spiropyran, whose closed-ring, hydrophobic SP isomer transforms into a highly polar, open-ring merocyanine (MC) form upon exposure to ultraviolet light, whereas the reverse reaction can be induced by visible light ${ }^{12-14}$. Moreover, this reversible isomerization can be triggered by a variety of other external stimuli, such as acids and bases, metal ions, temperature, redox potential and mechanical force. The isomerization process, however, entails a large change in the conformation of the molecule-an obstacle as far as efficient isomerization within solid-state materials is concerned. In order to efficiently switch, spiropyran molecules require conformational freedom, which usually is not available within the densely packed arrays of molecules in the crystalline state.

An attractive way to satisfy this conformational freedom requirement would be to harbour the spiropyran moieties within the nanopores of porous aromatic frameworks (PAFs)-a recently introduced ${ }^{15-17}$ family of microporous polyphenylene materials. This strategy could not only enable efficient isomerization of spiropyran in the solid state, but it would also allow for the control of the beneficial properties of PAFs (such as storage of gaseous cargo) using external stimuli. Here we describe two strategies (Fig. 1) to construct stimuli-responsive nanoporous
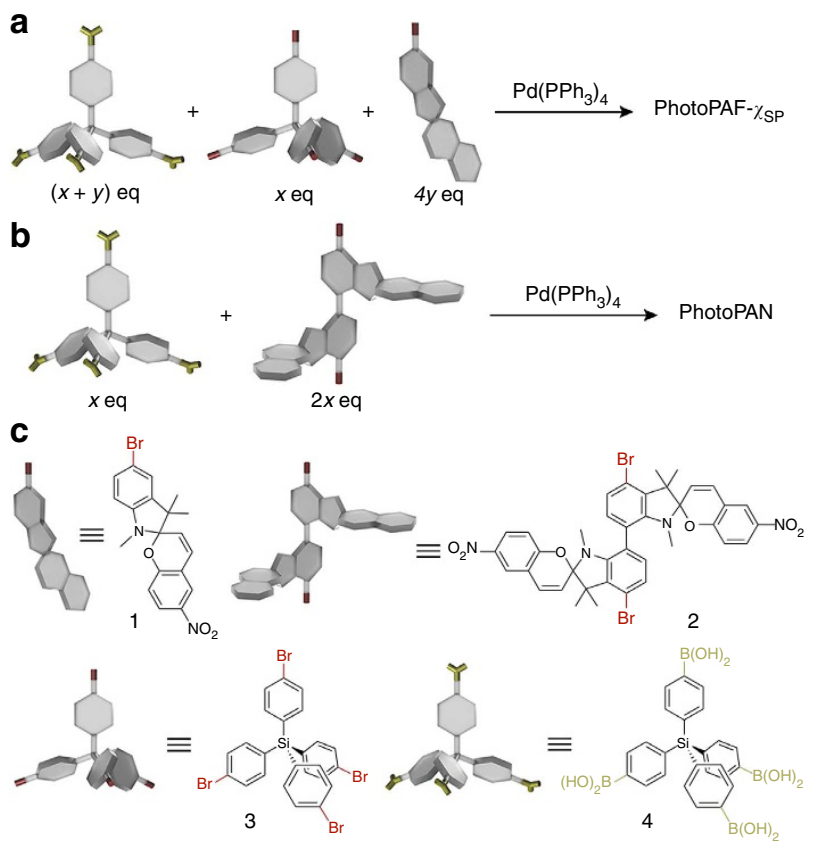

Figure 1 | Two strategies to prepare nanoporous materials incorporating spiropyran. (a) Preparation of photoresponsive porous aromatic framework (PhotoPAF). (b) Preparation of photoresponsive porous aromatic network (PhotoPAN). (c) Structural formulae of the building blocks. materials based on spiropyran: the first approach is based on a three-component Suzuki coupling reaction involving (i) a rigid, tetratopic boronic acid 4, (ii) a rigid, tetratopic bromide 3 , and (iii) a photoresponsive, monotopic bromide 1 . We refer to the products of this route as PhotoPAF- $\chi_{\mathrm{SP}}$ (photoresponsive porous aromatic framework), where $\chi_{\mathrm{SP}}$ indicates the extent of spiropyran loading. Second, we consider a two-component coupling between the boronic acid 4 and a rigid photoresponsive ditopic bromide 2 -here, the resulting material is found to have a significantly more flexible structure, hence, we refer to it as photoresponsive porous aromatic network or PhotoPAN. Both types of materials-PhotoPAFs and PhotoPAN-exhibit a variety of interesting properties, including light-controlled capture and release of metal ions, examples of reversible photochromism and acidochromism of spiropyran under solvent-free conditions, as well as reversible chromism induced by solvation/desolvation.

\section{Results}

Synthesis of nanoporous materials incorporating spiropyran. Both PhotoPAFs and PhotoPAN were synthesized via Pd-catalysed Suzuki cross-coupling reactions (see Supplementary Figs 128 and Supplementary Methods for the synthesis and characterization of all small molecules). In a typical procedure, a single-neck round-bottom flask was charged with a mixture of bromide and boronic acid precursors, $\mathrm{K}_{2} \mathrm{CO}_{3}$ and a catalytic amount of $\mathrm{Pd}\left(\mathrm{PPh}_{3}\right)_{4}$ were added, and the reactants were heated under a blanket of nitrogen in a 1:1 tetrahydrofuran (THF)-water solvent mixture at $75^{\circ} \mathrm{C}$ for 2 days. In the case of PhotoPAFs, precursors 1, 3 and 4 were used in ratios such that the total number of bromide groups (in $\mathbf{1}$ and $\mathbf{3}$ ) was equal to the number of boronic acid functionalities (in 4; see Fig. 1; we used Si-based, as opposed to C-based, building blocks $\mathbf{3}$ and $\mathbf{4}$ as they are easily prepared from commercially available starting materials ${ }^{18,19}$ ). For PhotoPAN, a 2:1 mixture of precursors $\mathbf{2}$ and 4 was used (Fig. 1). The solids that precipitated during the reaction were collected, washed extensively with water, acids, bases and various organic solvents, and finally dried in a vacuum oven (see Methods for detailed procedures). We employed a variety of characterization techniques to verify successful incorporation of spiropyran into, and to quantify its loading $\left(\chi_{\mathrm{SP}}\right)$ within the nanoporous materials (we define $\chi_{\mathrm{SP}}$ as the molar ratio of spiropyran in PhotoPAFs to the total ratio of aryl groups originating from starting materials 1 and 3$)$. $\chi_{\mathrm{SP}}$ values were determined by correlating weight percentages of carbon, hydrogen and nitrogen to the composition of the feed mixture as described in Methods. As expected, we found that $\chi_{\mathrm{SP}}$ was proportional to the percentage of $\mathbf{1}$ in the initial mixture; however, it was on average only half of the spiropyran content expected from the composition of the feed. This result can be understood based on the fact that $\mathbf{3}$ has four reactive groups; once one of them reacts, the molecule becomes incorporated in the growing network and the remaining three groups are more likely to react with the $-\mathrm{B}(\mathrm{OH})_{2}$ groups, which are also part of the existing polymer.

Successful coupling reaction as well as the presence of spiropyran in the final material was confirmed by infrared spectroscopy. We followed the progress of the reaction by monitoring the intensity of boronic acid 4's O-H stretching vibrations (the intense band centered at $\sim 3,400 \mathrm{~cm}^{-1}$ ). A nearly complete disappearance of absorption in this region in PhotoPAN and a variety of PhotoPAFs (Supplementary Fig. 29) confirms the high yield of the $\mathrm{C}-\mathrm{C}$ coupling reaction (residual absorption can be attributed to unreacted boronic acid or to the presence of small amounts of water in the nanopores). The presence and loading of 
spiropyran, on the other hand, are best appreciated by the peak at $\sim 1,340 \mathrm{~cm}^{-1}$, which is due to the symmetric stretching mode of the $\mathrm{NO}_{2}$ group (see Supplementary Fig. 29b) ${ }^{20}$.

To further demonstrate the successful incorporation of spiropyran, we performed ${ }^{1} \mathrm{H}$ and ${ }^{13} \mathrm{C}$ solid-state nuclear magnetic resonance (NMR) studies. ${ }^{1} \mathrm{H}$ magic angle spinning (MAS) NMR spectrum of PhotoPAF-3.6\%, for example, shows a major peak centered at $\delta=8.2$ p.p.m., in addition to a smaller one at $\sim 2$ p.p.m. (Supplementary Fig. 30a). The former can be attributed to protons bound to unsaturated carbon atoms, whereas the latter is due to the spiropyran's $\mathrm{CH}_{3}$ protons (and the residual solvent often observed ${ }^{15,21,22}$ in solid-state NMR experiments; we also observed a small peak in this region for a PAF lacking any spiropyran-cf Supplementary Fig. 30d). As expected, the upfield peak significantly increased in PhotoPAN, as shown in Supplementary Fig. 30b; interestingly, integrating the $\sim 8$ and $\sim 2$ p.p.m. signals gives the ratio of $\sim 1.1: 1$, very similar to the 11:9 stoichiometric ratio of the protons attached to unsaturated versus saturated carbons. Similar trends were found in ${ }^{13} \mathrm{C}$ spectra, whereby PhotoPAF-3.6\% and PhotoPAN showed increasing peaks in the $20-30$ p.p.m. region (diagnostic of the spiropyran's methyl groups, see right panel of Supplementary Fig. 30). Finally, the varying amounts of spiropyran were verified by simultaneous thermal gravimetric analysis (TGA)-differential scanning calorimetry (DSC) measurements (Supplementary Fig. 31). Both mass loss (TGA) and differential heat flux (DSC) showed two-step profiles, with a major change at $\sim 550^{\circ} \mathrm{C}-\mathrm{a}$ signature of the decomposition of the polyphenylene network ${ }^{15}$ accompanied by a minor one at $\sim 350^{\circ} \mathrm{C}$. This latter transition can be attributed ${ }^{23}$ to the loss of spiropyran-indeed this transition is absent from spiropyran-free PAF, and it becomes increasingly pronounced as the amount of the switch increases (see Supplementary Fig. 31).

Finally, we performed several control experiments to verify whether the spiropyran moieties are indeed incorporated covalently within the host PAFs, as opposed to 1 being trapped in the nanopores during the synthesis of the frameworks. First, to demonstrate the activity of the aryl bromide $\mathbf{1}$ in the Suzuki crosscoupling reaction, we attempted a model coupling between $\mathbf{1}$ and phenylboronic acid under conditions identical to those employed in the preparation of PhotoPAFs. Successful synthesis and characterization of the coupling product is described in Supplementary Methods (see also Supplementary Figs 26-28). Second, as-prepared PhotoPAFs were washed multiple times (see detailed procedure in Methods) to ensure the removal of all residual small molecules. Finally, we performed a two-component coupling involving stoichiometric amounts of $\mathbf{3}$ and $\mathbf{4}$ in the presence of the parent spiropyran $\left(1^{\prime}, 3^{\prime}\right.$-dihydro- $1^{\prime}, 3^{\prime}, 3^{\prime}$ trimethyl-6-nitrospiro[2H-1-benzopyran-2,2'-(2H)-indole])-that is, 1 lacking the bromide group. Following the standard washing procedure, we found no signature of the spiropyran group in any of the characterization techniques described above.

Spiropyran isomerization triggered by desolvation and structural collapse. Figure 2 shows typical optical and electron micrographs of our nanoporous materials (see Supplementary Figs 32 and 33 for additional images). As evident from the electron micrographs, the structures of both PhotoPAFs and PhotoPAN lack long-range order, in agreement with previous reports $^{15,24,25}$ on PAFs obtained by irreversible C-C coupling reactions. Still, the porous nature of these materials can easily be discerned by transmission electron microscopy (TEM): focusing on the edge of the sample revealed the presence of $\sim 1 \mathrm{~nm}$ nanopores (Fig. 2d). Interestingly, inspection of the samples using electron microscopy methods revealed an unexpected difference
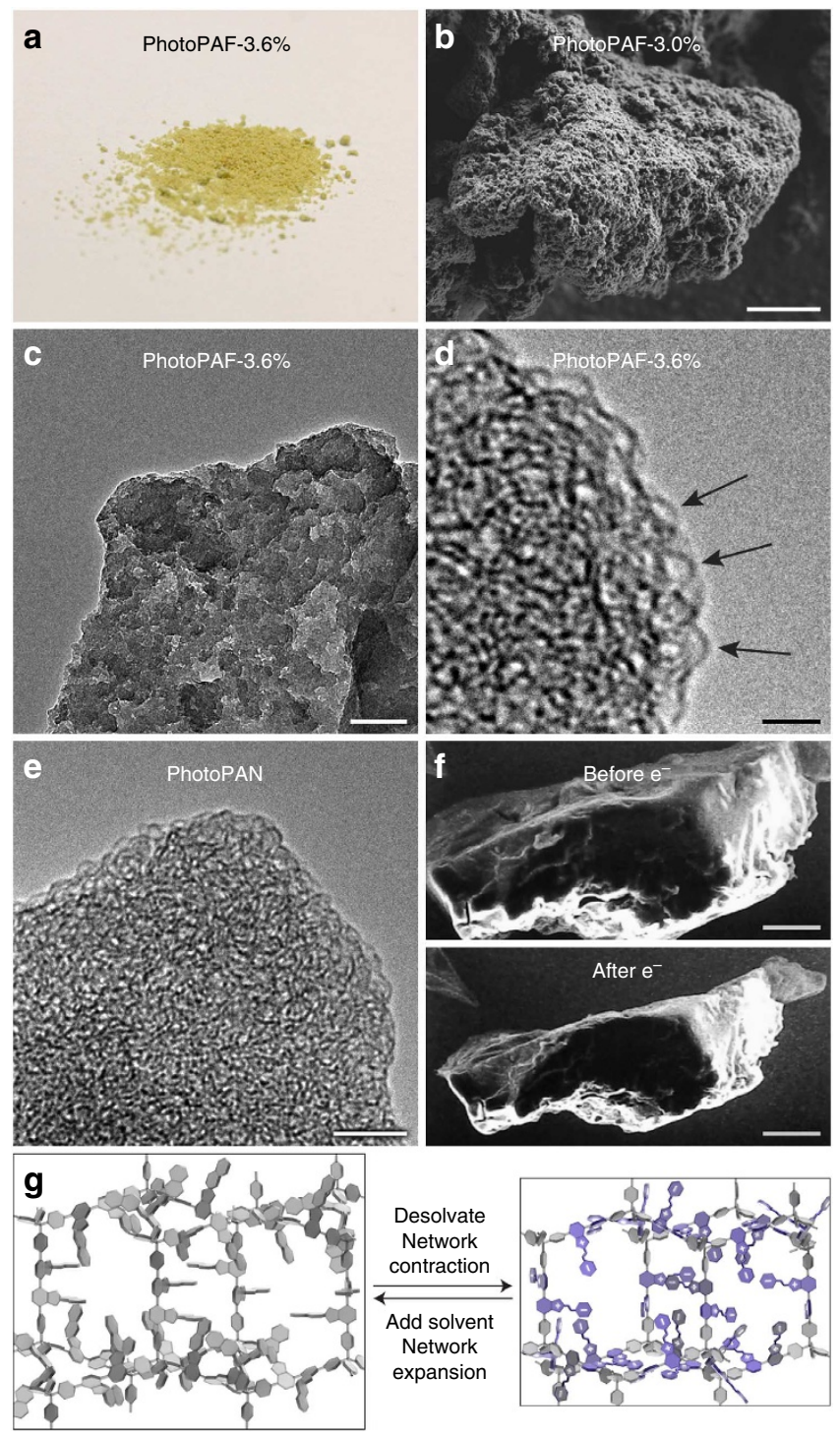

Figure 2 | Structures of nanoporous materials incorporating spiropyran.

(a) Photograph of a macroscopic sample of photoresponsive porous aromatic framework (PhotoPAF)-3.6\% ( 100 mg). (b) Scanning electron microscopy image of PhotoPAF-3.0\%. (c) Transmission electron microscopy (TEM) image of PhotoPAF-3.6\% at a low magnification. (d) TEM image of PhotoPAF-3.6\% at a high magnification. Arrows indicate entities that are likely individual nanopores. (e) TEM image of photoresponsive porous aromatic network (PhotoPAN). (f) Structural collapse of PhotoPAN upon prolonged exposure to the electron beam (see also Supplementary Movies 1-3). (g) Schematic representation of PhotoPAN network contraction/expansion accompanied by spiropyran ring opening/closing as a result of desolvation/solvation. Scale bars correspond to $5 \mu \mathrm{m}$ (b), $100 \mathrm{~nm}$ (c), $2 \mathrm{~nm}$ (d), $5 \mathrm{~nm}$ (e) and $50 \mu \mathrm{m}$ (f).

in the mechanical properties of these two types of materials: when exposed to the electron beam under reduced $\left(\sim 10^{-5} \mathrm{mBar}\right)$ pressure typical for the operation of electron microscopes, the structure of PhotoPAN collapsed (cf scanning electron microscopy (SEM) images in Fig. 2f; see also Supplementary Movies 1-3), whereas PhotoPAFs remained unaffected. This collapse can be attributed to the removal of residual solvent molecules from the nanopores of PhotoPAN; the absence of analogous structural changes in PhotoPAFs is likely due to the more rigid nature of their pores (lacking the 2 'spacers'). 
The low pressure under which electron microscopes operate, however, was not essential to induce the structural collapse of PhotoPAN: we observed a shrinking of the grains even during filtration on a Büchner funnel (overall, drying and exposure to the electron beam decreased the volume of the grains by $\sim 80 \%$, comparable to the free volume of other ${ }^{15,26,27}$ porous frameworks). More remarkably, this shrinking was accompanied by a pronounced change in colour from dark yellow to deep blue. The blue colour is the characteristic of MC: the metastable, openring isomer of spiropyran (see Fig. 3a). As previously established $^{28-31}$, the normally metastable MC moieties can stabilize one another by interacting via a combination of electrostatic, dipolar and $\pi-\pi$ interactions ${ }^{32}$; this stabilization can trigger spontaneous $\mathrm{SP} \rightarrow \mathrm{MC}$ transition when the closed-ring SP moieties are brought in close proximity ${ }^{33,34}$. The uniqueness of PhotoPAN lies also in the fact that the transition is reversible: when immersed in various solvents (hexane, toluene,

a

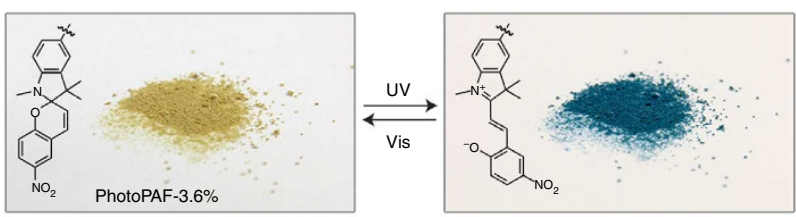

b

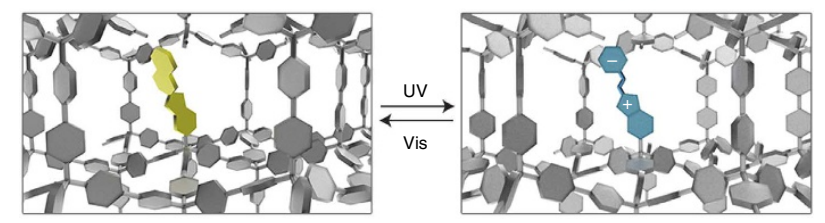

C
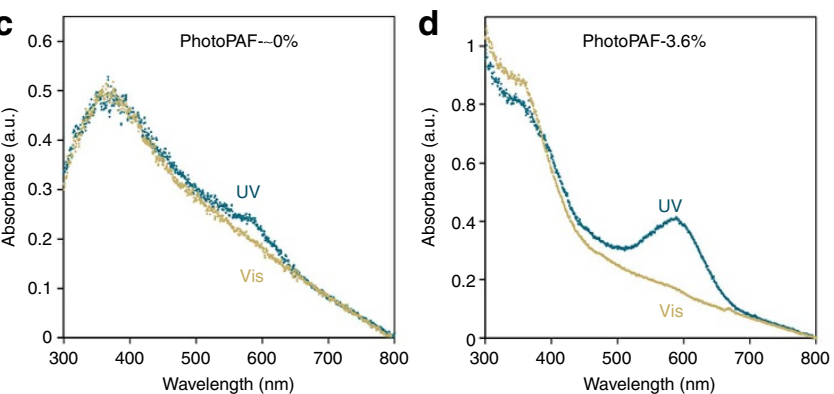

e
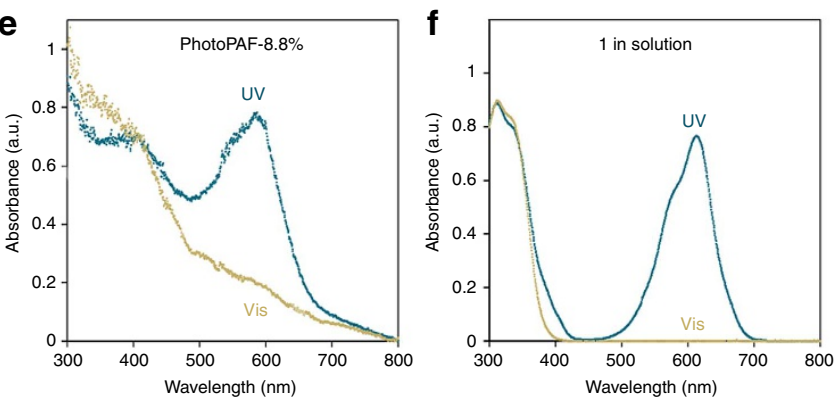

Figure 3 | Optical properties of PhotoPAFs. (a) Visual changes to PhotoPAF-3.6\% upon exposure to low-intensity ultraviolet light.

(b) Schematic representation of efficient isomerization of spiropyran inside the nanopores. (c-e) Ultraviolet-visible spectra of suspensions of PhotoPAFs (in 1:1 $\mathrm{PhMe}-\mathrm{CHCl}_{3}$ ) containing different amounts of spiropyran before and after ultraviolet irradiation. PhotoPAF 0\% (c) indicates a sample with a nitrogen content below the detection limit of the available elemental analyser. Integration of the merocyanine peak allows us to determine the composition of the sample as PhotoPAF-0.5\%. (f) Ultraviolet-visible spectra of a toluene solution of $\mathbf{1}$ before and after ultraviolet irradiation. Vis, visible. dimethylformamide, methanol, etc.), the particles swelled (Fig. 2g) and regained their original dimensions, whereas their colour faded. Both processes are fast: PhotoPAN resolvated and the colour faded within only a few seconds, whereas shrinking and colouration took less than a second. Moreover, as the transition was not induced by light, the spiropyran switch exhibited excellent reversibility: even after performing 200 solvation-desolvation cycles, we saw no signs of degradation of the switch.

The absence of the MC form within the solvated PhotoPAN was confirmed by ultraviolet-visible spectroscopy: we observed no peak at $\sim 550 \mathrm{~nm}$ in optical spectra of the PhotoPAN suspensions in solvents of different polarities (for example, acetonitrile, THF, toluene). At the same time, the formation of MC could be induced by exposing the samples to lowintensity ultraviolet radiation $\left(\lambda=365 \mathrm{~nm} ; I=0.7 \mathrm{~mW} \mathrm{~cm}^{-2}\right.$; Supplementary Fig. 34), and the resulting blue suspensions faded spontaneously with kinetics characteristic of those of smallmolecule spiropyrans in hydrophobic solvents-in other words, 'normal' photochromism of spiropyran was observed in all solvents investigated. This finding is in contrast to previous reports on small-molecule spiropyrans, where the equilibrium between the SP and MC isomers was strongly dependent on the polarity of the solvent ${ }^{32,35}$ (solvatochromism; for example, the relatively polar acetonitrile typically stabilizes the MC form). We find, however, that the aromatic character of the network backbone has a 'buffering' effect on the photoswitch, favouring the closed-ring SP isomer irrespective of the solvent.

Efficient isomerization of spiropyran in the solid state. Although spiropyran within the solvent-free PhotoPAN was permanently 'frozen' in the MC state, its solid-state photoisomerization could readily be accomplished when within the nanopores of the more rigid PhotoPAFs. As Fig. 3a shows, for example, when the yellow powder of PhotoPAF-3.6\% was exposed to low-intensity ultraviolet light, it turned deep blue within $\sim 3 \mathrm{~min}$, indicative of $\mathrm{SP} \rightarrow \mathrm{MC}$ isomerization (see also Supplementary Fig. 35). Such efficient photoisomerization at room temperature and under solvent-free conditions is, to our knowledge, unprecedented: although solid-state isomerization of other molecular photoswitches has been reported ${ }^{36-38}$, the significant conformational difference between the closed- and the open-ring isomers of spiropyran has effectively prohibited the long-sought ${ }^{39-41}$ photoisomerization of this class of molecules in the solid state. The MC peak was clearly visible at $\sim 590 \mathrm{~nm}$ in the optical spectra recorded on suspensions of different PhotoPAFs; in addition, as Fig. 3c-e shows, ultraviolet-visible spectroscopy served as yet another way to demonstrate the control over spiropyran loading: the $\sim 590 \mathrm{~nm}$ peak grew with increasing amounts of the photoswitch within the PhotoPAFs. The MC-functionalized PhotoPAFs slowly (within several hours) faded as MC isomerized back to SP in a reaction that could be accelerated (to around a minute) with visible light. These photoirradiation experiments confirmed the rigid nature of PhotoPAFs: we saw (by SEM) no noticeable differences in the size and shape of the samples before and after exposure to ultraviolet and then visible light.

Covalent immobilization of spiropyran units prevents them from interacting with one another, which is important in the context of reversible isomerization. As previously established ${ }^{42,43}$, irreversible reactions between excited spiropyran molecules are the primary cause of fatigue - that is, gradual degradation of a molecular switch as a result of reversible photoisomerization. This 'bimolecular' pathway, however, is greatly inhibited in PhotoPAFs; indeed, we found that deoxygenated (purged 
with argon) PhotoPAF-3.6\% was virtually fatigue-free and no indication of photodegradation could be seen even after performing 100 switching cycles (one cycle $=1 \mathrm{~min}$ of ultraviolet, $I=0.7 \mathrm{~mW} \mathrm{~cm}^{-2}$, followed by $1.5 \mathrm{~min}$ of visible light, fluorescent bulb $I=1.0 \mathrm{~mW} \mathrm{~cm}^{-2}$ ). In air, however, the 'monomolecular' (oxygen-dependent) degradation pathway was still functional ${ }^{44,45}$, leading to significant fatigue effects as reported in prior literature ${ }^{46}$.

Photoswitchable fluorescence. An interesting feature of the spiropyran switch is that while its closed-ring, SP isomer is practically non-fluorescent, MC shows an intense red emission ${ }^{47,48}$. Consequently, being able to reversibly photoisomerize under solvent-free conditions, PhotoPAFs showed unique solid-state photoswitchable fluorescence, as shown in Fig. $4 \mathrm{a}$. The intense red emission not only confirms a successful $\mathrm{SP} \rightarrow \mathrm{MC}$ isomerization, but also implies that the MC units within the ultraviolet-irradiated PhotoPAFs do not interact with one another; we note that MC-MC stacking leads to fluorescence quenching as reported earlier in the literature ${ }^{49}$, and as manifested by the properties of solid-state PhotoPAN, which, despite featuring spiropyran in the MC form without any ultraviolet irradiation, showed no measurable emission. Figure $4 \mathrm{~b}$ shows photoswitchable fluorescence of a single particle of PhotoPAF-3.6\% investigated using fluorescence microscopy (see also Supplementary Movie 4), along with a typical emission spectrum recorded on the same particle $\left(\lambda_{\max }=660 \mathrm{~nm}\right)$. We also employed confocal fluorescence microscopy to verify that no phase separation occurs during the synthesis of PhotoPAFs (phase separation could take place if aryl bromides 1 and 3 reacted with the boronic acid $\mathbf{4}$ with vastly different kinetics). Optical sections acquired over a range of specimen planes (for example, Fig. 4c) show no preferential accumulation of the fluorescent component at the centre or at the periphery of the grains.

Acidochromism induced by gaseous signals. Next, we hypothesized that the switchable moieties located within the nanopores could be actuated by gaseous signals (Fig. 5a; in solution, MC's phenoxide anion undergoes protonation upon the addition of strong acids, resulting in a yellow-coloured $\mathrm{MCH}^{+}$ species $^{50}$; see also Supplementary Fig. 34). To test our hypothesis, we passed gaseous $\mathrm{HCl}$ through a pad of solid PhotoPAN and observed a slow colour change from deep blue to bright yellow (Fig. 5b). The seemingly complete disappearance of the initial dark colour suggests a high yield of the $\mathrm{MC} \rightarrow \mathrm{MCH}^{+}$ conversion. The protonation reaction was reversible: gaseous $\mathrm{NH}_{3}$ abstracted protons from $\mathrm{MCH}^{+}$and re-generated the deeply coloured MC-functionalized PhotoPAN. Interestingly, the two processes differed significantly in their kinetics: although protonation occurred gradually over the course of ca $5 \mathrm{~min}$, deprotonation was nearly instantaneous (see Supplementary Movie 5). Given that the van der Waals radii of $\mathrm{HCl}$ and $\mathrm{NH}_{3}$ are very similar, such significant discrepancies should be attributed to the differences in reaction kinetics rather than different diffusion coefficients. Overall, these results demonstrate that despite its collapsed structure, MC-rich PhotoPAN still comprises nanopores large enough to allow for transport of small molecules. An interesting consequence of the alternate infusion of $\mathrm{HCl}$ and $\mathrm{NH}_{3}$ is the formation of ammonium chloride in the nanopores of PhotoPAN. Each protonation-deprotonation cycle gives rise to a maximum of one equivalent of $\mathrm{NH}_{4} \mathrm{Cl}$ with respect to the number of MC units. An intriguing question that we will address separately concerns the nature of $\mathrm{NH}_{4} \mathrm{Cl}$ formed within the nanopores, whose sizes likely prohibit the formation of a crystalline phase of the salt.

Light-controlled uptake and release of metal ions. Finally, we considered the possibility of employing PhotoPAN as a lightcontrolled sponge for metal ions. Here, we take advantage of the vastly different affinities of the SP and MC isomers to metal cations: although SP lacks appreciable complexing ability, MC binds metal ions via the phenoxide anions in a 2:1 stoichiometry ${ }^{51,52}$. To demonstrate the proof-of-concept, we chose $\mathrm{Cu}^{2+}$ because of its intense absorption of light in the visible region

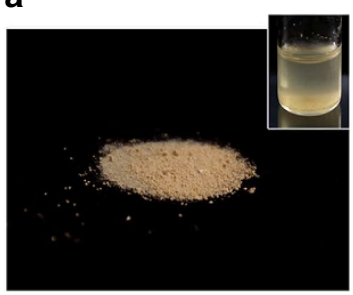

PhotoPAF-3.6\%

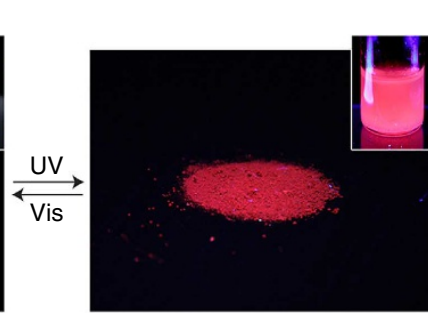

b

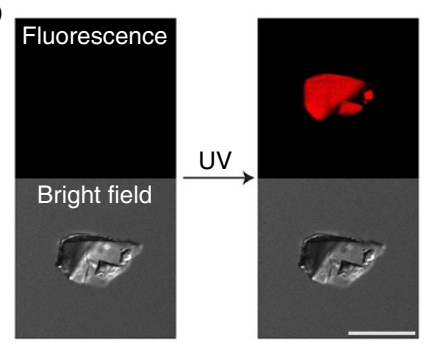

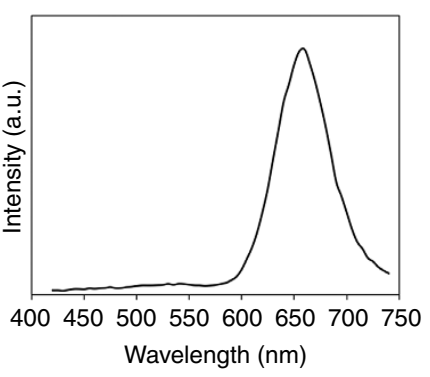

Wavelength $(\mathrm{nm})$

C
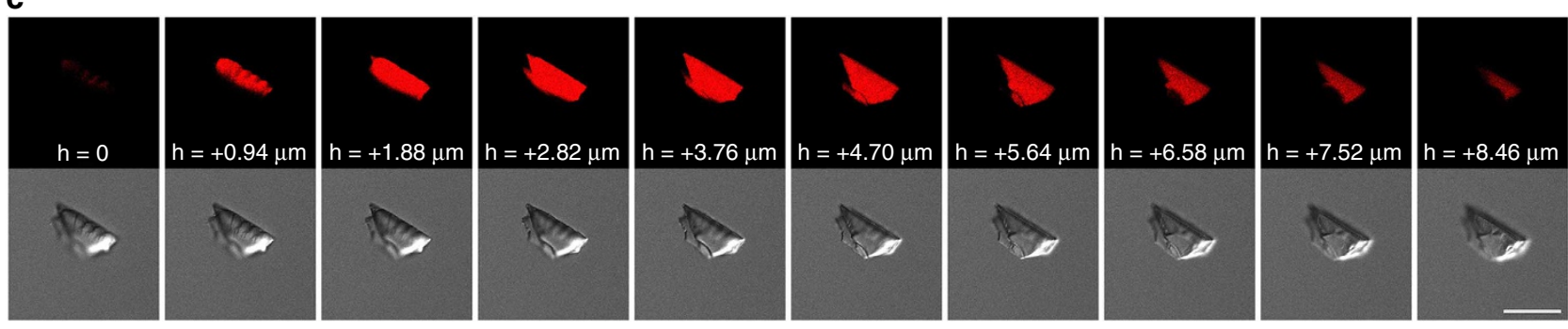

Figure 4 | Photoswitchable fluorescence in PhotoPAFs. (a) Visual changes to the sample in the solid state and in a toluene suspension (insets) as a result of ultraviolet irradiation. Images on the left were taken under ambient light. Images on the right were taken in the dark under $365 \mathrm{~nm}$ illumination. (b) Using ultraviolet to 'turn on' fluorescence of a single PhotoPAF-3.6\% grain. Also shown is an emission spectrum (excitation wavelength $=405 \mathrm{~nm}$ ) of the same grain. (c) Confocal fluorescence micrographs of a ultraviolet-activated PhotoPAF-3.6\% grain focused on different planes along the optical axis of the microscope. Scale bars correspond to $20 \mu \mathrm{m}$. 
a

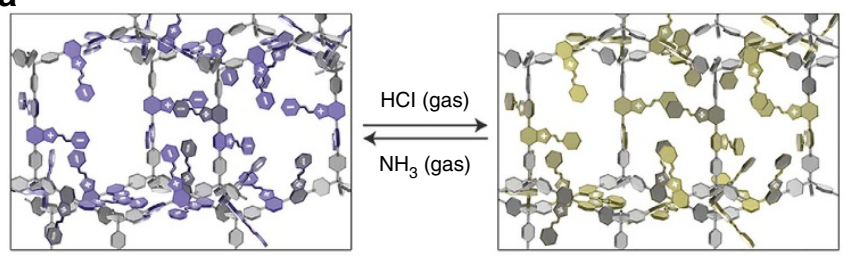

b

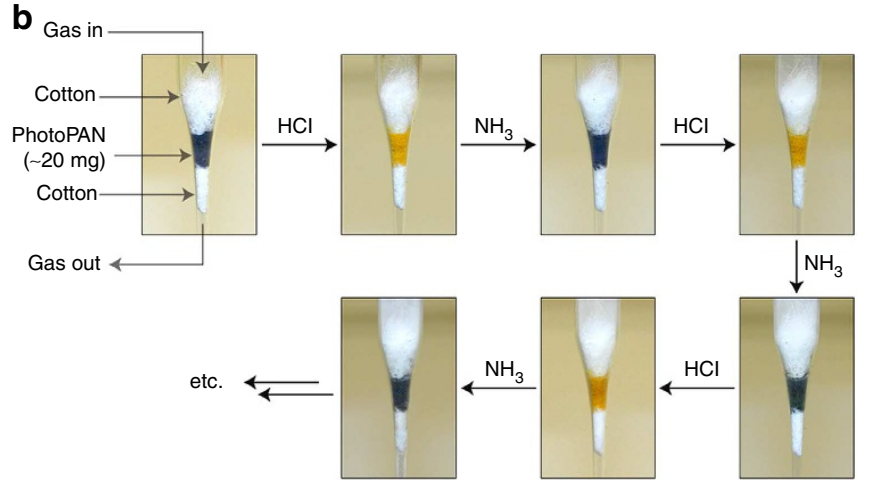

Figure 5 | Acidochromism of PhotoPAN. (a) Schematic representation of reversible protonation of PhotoPAN induced by gaseous $\mathrm{HCl}$ and $\mathrm{NH}_{3}$. (b) Experimental setup used to investigate solid-state acidochromism and demonstration of three cycles of protonation/deprotonation.

( $\lambda_{\max }=460 \mathrm{~nm}$ for $\mathrm{CuCl}_{2} \cdot 2 \mathrm{H}_{2} \mathrm{O}$ in acetonitrile). Six minutes of sonication with $5 \mathrm{mg}$ of PhotoPAN was sufficient to quantitatively remove $\mathrm{Cu}^{2+}$ from an acetonitrile solution of $0.4 \mathrm{mg}$ of $\mathrm{CuCl}_{2} \cdot 2 \mathrm{H}_{2} \mathrm{O}$ (Fig. $6 \mathrm{~b}, \mathrm{~d}$ ) in a process that involved $\mathrm{Cu}^{2+}$-induced SP $\rightarrow$ MC isomerization (Supplementary Fig. 36a; the ratio we used corresponded to a small excess of SP to $\mathrm{Cu}^{2+}$ ( 2.6-to-1; compared with the 2:1 stoichiometric ratio)). As a control experiment, we sonicated the same amount of the copper salt with $5 \mathrm{mg}$ of spiropyran-free PAF (that is, 'PhotoPAF-0\%'), and observed negligible absorption of $\mathrm{Cu}^{2+}$ even after $30 \mathrm{~min}$ of sonication (Supplementary Fig. 36b). Next, we exposed the $\mathrm{Cu}^{2+}$-soaked PhotoPAN to visible light $\left(I=1.0 \mathrm{~mW} \mathrm{~cm}^{-2}\right)$ and observed a gradual release of copper ions (Fig. 6c, d). PhotoPAFs containing different amounts of spiropyran behaved similarly to PhotoPAN. Although exposure to light induced a side reaction (the well-known ${ }^{5,54}$ visible light-induced decomposition of $\mathrm{CuCl}_{2}$ in acetonitrile) and the absorption of the final supernatant was lower than that of the initial solution (see Supplementary Fig. 36c), quantitative release of copper was confirmed by energy-dispersive X-ray spectroscopy (EDS), which showed the absence of copper in the final PhotoPAN.

\section{Discussion}

In summary, we developed two synthetic approaches to spiropyran-based nanoporous materials responsive to multiple types of external stimuli. The two approaches are complementary: one leads to structurally rigid PhotoPAFs-materials that feature efficient spiropyran isomerization and MC fluorescence photoswitching in the solid state. The more flexible PhotoPANs, on the other hand, display solid-state acidochromism, light-controlled trapping and release of metal ions from solution, as well as a novel type of chromism induced by solvation and desolvation of the network. These findings have many interesting implications. First, solid-state photoswitching between two isomers (that is, SP and $\mathrm{MC}$ ) differing vastly in their polarity is expected to affect the amount of gas (for example, $\mathrm{H}_{2}, \mathrm{CH}_{4}$ ) trapped in the nanopores, a
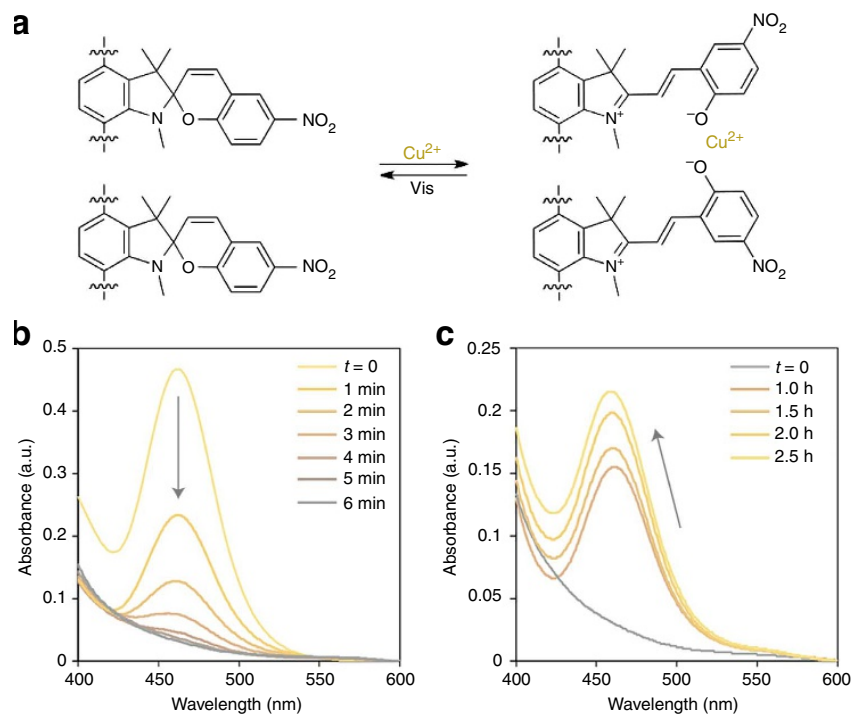

d

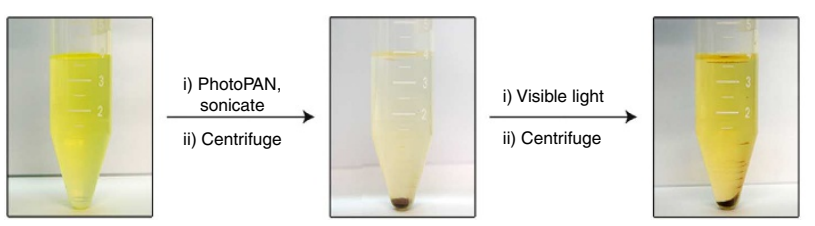

Figure 6 | lonochromism of PhotoPAN. (a) Reversible metal ion/lightinduced ring opening/closing. (b) Decrease in optical absorption of $\mathrm{Cu}^{2+}$ as a result of trapping inside PhotoPAN. (c) Increase in optical absorption of $\mathrm{Cu}^{2+}$ as a result of visible light-induced release. All spectra were recorded on supernatant after centrifugation. (d) Visual changes to the copper salt solution as the copper ions are removed from and then released back to the solution. All experiments shown here were performed using $5 \mathrm{mg}$ of PhotoPAN and $0.4 \mathrm{mg} \mathrm{CuCl} 2 \cdot 2 \mathrm{H}_{2} \mathrm{O}$.

and hence, it can enable the control of gas sorption using light. Second, the reversible capture and release of metal ions can be transduced into catalytic properties of PhotoPANs (for example, initial experiments show that $\mathrm{Cu}^{2+}$-functionalized PhotoPAN is a highly active catalyst for the hydrolysis of bromobenzenes). Third, our studies of acidochromism led to the realization that the nanopores of PhotoPANs can act as templates for the preparation of various materials, ammonium chloride being the first material prepared in these 'nanoflasks'. Finally, we note that our methodology (Fig. 1) is applicable to other functional nanoporous materials, and it allows for the incorporation of more than one type of functionality. In one direction that we are actively pursuing, a four-component Suzuki coupling leads to frameworks featuring both spiropyran and catalytic moieties: we are investigating the applicability of these materials as a novel family of light-controlled catalysts ${ }^{55}$.

\section{Methods}

General. Solution ${ }^{1} \mathrm{H}$ and ${ }^{13} \mathrm{C}$ NMR spectra were recorded at room temperature on Bruker 300 and $500 \mathrm{MHz}$ spectrometers (Bruker). Chemical shifts $(\delta)$ are reported in p.p.m.; multiplicities are indicated by 's' (singlet), 'd' (doublet), 't' (triplet), 'q' (quartet), 'quint' (pentet), 'm' (multiplet) or 'br' (broad). Coupling constants $(J)$ are reported in Hz. Spectra were referenced to residual chloroform ( ${ }^{1} \mathrm{H}: \delta=7.26$ p.p.m.; ${ }^{13} \mathrm{C}: \delta=77.00$ p.p.m.), dimethyl sulfoxide $\left({ }^{1} \mathrm{H}: \delta=2.50\right.$ p.p.m.; ${ }^{13} \mathrm{C}: \delta=39.52$ p.p.m.) or dichloromethane $\left({ }^{1} \mathrm{H}: \delta=5.32\right.$ p.p.m.; ${ }^{13} \mathrm{C}: \delta=53.84$ p.p.m.). High-resolution mass spectra were recorded at $60-70 \mathrm{eV}$ on a Waters Micromass Q-TOF spectrometer (ESI, Ar; Waters). Single crystal X-ray data for 1 were collected on a Bruker Appex2 KappaCCD diffractometer (Bruker; Mo K $\alpha$, $\lambda=0.71073 \AA$, graphite monochromator, $-10 \leq h \leq 10,-24 \leq k \leq 24$,

$-22 \leq l \leq 22$, frame scan width $=0.5^{\circ}$, scan speed $1.0^{\circ}$ per $20 \mathrm{~s}$, typical peak mosaicity $0.74^{\circ}, 42,225$ reflections collected, 3,870 independent reflections; 
$R$-int $=0.039$ ). The data were processed with Denzo-Scalepack. Structure of $\mathbf{1}$ was solved with SHELXS. Full matrix least-squares refinement based on $F^{2}$ with

SHELXL-97 on 295 parameters with no restraints gave final $R_{1}=0.0223$ (based on $\mathrm{F}^{2}$ ) for data with $I>2 \sigma(I)$ and $R_{1}=0.0269$ on 8,216 reflections, goodness-of-fit on $F^{2}=1.030$, largest electron density peak $=0.406 \AA^{-3}$, largest hole $=-0.263 \AA^{-3}$, see also Supplementary Data 1. Elemental analyses were performed on a Thermo Scientific FlashEA 1112 organic elemental analyser (Thermo Scientific). Fourier transform-infrared spectroscopy was carried out on a Nicolet 380 infrared spectrometer at $4 \mathrm{~cm}^{-1}$ resolution. The spectra were recorded on finely ground samples $(\sim 0.2 \mathrm{mg})$ premixed with $\sim 20 \mathrm{mg}$ of $\mathrm{KBr}$ (Fourier transform-infrared grade) and pressed into $7-\mathrm{mm}$ pellets using a manual hydraulic press (Specac). TGA and DSC were performed on an SDT Q600 V8.3 Build 101 simultaneous DSC-TGA instrument operating in dry air in the temperature range $25-800{ }^{\circ} \mathrm{C}$, at a heating rate of $20^{\circ} \mathrm{C} \mathrm{min}^{-1}$. SEM was performed on an ULTRA 55 field emission SEM (Carl Zeiss Microscopy, LLC; Carl Zeiss) as well as on a SUPRA 55VP field emission SEM (Carl Zeiss Microscopy, LLC), both operating at $5 \mathrm{kV}$. TEM was performed on a CM120 Super Twin TEM (Philips) operating at $120 \mathrm{kV}$. EDS measurements were carried out on a SUPRA 55VP field emission SEM equipped with an Oxford Instruments EDS detector. Ultraviolet-visible spectra were recorded on a Shimadzu UV-3600 spectrometer (Shimadzu). All ultraviolet-visible spectra of PhotoPAFs/PhotoPAN are averages of at least three individual spectra. For ultraviolet irradiation, we used a 4 -W

hand-held UV lamp (UVP, LLC; model number UVGL-25; light intensity $\sim 0.7 \mathrm{~mW} \mathrm{~cm}^{-2}$ ) or a 100-W UV lamp (UVP, LLC; model number B-100AP; light intensity $\sim 10 \mathrm{~mW} \mathrm{~cm}^{-2}$ ). As a visible light source, we used a fluorescent bulb (light intensity $\sim 1.0 \mathrm{~mW} \mathrm{~cm}{ }^{-2}$ ). Confocal fluorescence imaging and spectral imaging were performed on an IX81-based Olympus FluoView 1000D laser confocal scanning microscope (Olympus), equipped with a spectral scanning system and two independent laser scanners. To induce SP ring opening, we used light of $\lambda=405 \mathrm{~nm}$. All pictures were collected using a $655-755 \mathrm{~nm}$ emission filtre following excitation with a $635-\mathrm{nm}$ laser. To collect emission spectra, we used an excitation wavelength of $405 \mathrm{~nm}$.

Preparation of PhotoPAF. A single-neck, round-bottom flask with a magnetic stirrer was charged with $4\left(51.2 \mathrm{mg} ; 0.1 \mathrm{mmol} ; 0.4 \mathrm{mmol}\right.$ of $\left.-\mathrm{B}(\mathrm{OH})_{2}\right), 3(58.7 \mathrm{mg}$; $0.09 \mathrm{mmol} ; 0.36 \mathrm{mmol}$ of $-\mathrm{Br}), 1$ ( $16.05 \mathrm{mg} ; 0.04 \mathrm{mmol} ; 0.04 \mathrm{mmol}$ of $-\mathrm{Br})$, $\mathrm{Pd}\left(\mathrm{PPh}_{3}\right)_{4}(11.6 \mathrm{mg} ; 0.01 \mathrm{mmol})$ and potassium carbonate $(138.2 \mathrm{mg} ; 1 \mathrm{mmol})$. The flask was fitted with a reflux condenser closed with a septum. The reaction mixture was degassed and filled with $\mathrm{N}_{2}$, and the procedure was repeated two more times. To the stirred solid mixture under a nitrogen atmosphere, $\mathrm{H}_{2} \mathrm{O}(1 \mathrm{ml})$ was added followed by THF $(1 \mathrm{ml})$, and the reaction mixture was refluxed for 2 days at $75^{\circ} \mathrm{C}$ (an additional $1 \mathrm{ml}$ of THF was added after $16 \mathrm{~h}$ of reflux). After cooling to room temperature, the reaction mixture was slowly quenched with a concentrated aqueous solution of $\mathrm{HCl}(1 \mathrm{ml})$, and the solid residue was filtered. The residue was then washed thoroughly with $\mathrm{H}_{2} \mathrm{O}(50 \mathrm{ml})$, aqueous $\mathrm{NaOH}$ solution $(c=1 \mathrm{M}$; $50 \mathrm{ml})$, hot $\mathrm{H}_{2} \mathrm{O}(70 \mathrm{ml})$, hot THF $(75 \mathrm{ml})$ and hot $\mathrm{CHCl}_{3}(75 \mathrm{ml})$. The residue was dried under reduced pressure at $75^{\circ} \mathrm{C}$ to yield pure PhotoPAF $(60 \mathrm{mg})$. The procedure described above uses a spiropyran feed ratio, $\alpha_{\mathrm{SP}}$, of $10 \%$. For other PhotoPAFs, relative amounts of $\mathbf{1}$ and $\mathbf{3}$ were varied. Actual content of spiropyran in the resulting materials was calculated from elemental analysis data (see Methods).

Preparation of PhotoPAN. An oven-dried, round-bottom flask with magnetic stirrer was charged with $4(51.2 \mathrm{mg} ; 0.1 \mathrm{mmol}), 2(160.1 \mathrm{mg} ; 0.2 \mathrm{mmol})$ and $\mathrm{Pd}\left(\mathrm{PPh}_{3}\right)_{4}(34.7 \mathrm{mg}, 0.03 \mathrm{mmol})$. After adding an aqueous solution of $\mathrm{K}_{2} \mathrm{CO}_{3}$ $(c=1 \mathrm{M} ; 1 \mathrm{ml} ; 1 \mathrm{mmol})$, the flask was quickly fitted with a reflux condenser connected to a Schlenk line via a septum. The reaction mixture was degassed, filled with $\mathrm{N}_{2}$ and the procedure was repeated two more times. THF $(4 \mathrm{ml})$ was added through the septum over the condenser and the reaction mixture was stirred at the reflux temperature $\left(75^{\circ} \mathrm{C}\right)$ under a nitrogen atmosphere for $24 \mathrm{~h}$. After cooling to room temperature, the reaction mixture was slowly quenched with a concentrated aqueous solution of $\mathrm{HCl}(1 \mathrm{ml})$, and the solid residue was filtered. The solid residue was then washed thoroughly with $\mathrm{H}_{2} \mathrm{O}(75 \mathrm{ml})$, aqueous $\mathrm{NaOH}$ solution $(c=1 \mathrm{M} ; 75 \mathrm{ml})$, hot $\mathrm{H}_{2} \mathrm{O}(75 \mathrm{ml})$, hot THF $(100 \mathrm{ml})$ and hot $\mathrm{CH}_{2} \mathrm{Cl}_{2}$ $(100 \mathrm{ml})$. The solid product was then suspended in $\mathrm{CH}_{2} \mathrm{Cl}_{2}(20 \mathrm{ml})$, sonicated for $1 \mathrm{~min}$ and filtered to get PhotoPAN $(150 \mathrm{mg})$, which was then dried under reduced pressure at $75^{\circ} \mathrm{C}$

Calculation of the degree of PhotoPAF substitution. To ensure the formation of infinite networks, we use equimolar amounts of the $-\mathrm{B}(\mathrm{OH})_{2}$ and the $-\mathrm{Br}$ groups in the starting materials, the latter originating from both tetrakis(4-bromophenyl) silane 4 and 5'-bromospiropyran 1-see Supplementary Fig. 37, where the spiropyran feed ratio, $\alpha_{\mathrm{SP}}$, is defined as the molar ratio of bromospiropyran to the total number of bromoaryl moieties in the starting mixture:

$$
\alpha_{\mathrm{SP}}=\frac{a}{a+4 b} .
$$

We find that in all cases, the content of spiropyran in the final PAFs is lower than that in the initial ('feed') mixture. Let us define the content of spiropyran in the final PAFs, $\chi_{\mathrm{SP}}$, as the quotient of the number of spiropyran units and the total number of benzene rings originating from $\mathrm{Ar}-\mathrm{Br}$ :

$$
\chi_{\mathrm{SP}}=\frac{x}{x+4 b},
$$

where $x<a$. To calculate $\chi_{\mathrm{SP}}$, we rely on elemental analysis data, and make an assumption that both (silanetetrayltetrakis(benzene-4,1-diyl))tetraboronic acid (3) and $\mathbf{4}$ are quantitatively incorporated into the forming PAFs (this assumption is reasonable as each of these substrates has as many as four reactive groups). Let us denote the fraction of residual (unreacted) boronic acid groups in PAFs as $y$, and the fraction of residual bromine groups as $z$ (see Supplementary Fig. 37). Weight percentage of carbon in the final material, $\% C$ :

$$
\begin{aligned}
\% C= & \frac{m_{\mathrm{a}, C}\left[19 x-24 \alpha_{\mathrm{SP}}\right]}{\left\{m_{\mathrm{a}, C}\left[19 x-24 \alpha_{\mathrm{SP}}\right]+m_{\mathrm{a}, H}\left[2 y+17 x-16 \alpha_{\mathrm{SP}}\right]+m_{\mathrm{a}, O}(2 y+3 x)\right.} \\
& \left.+m_{\mathrm{a}, N} 2 x+m_{\mathrm{a}, B} y-m_{\mathrm{a}, S i} \alpha_{\mathrm{SP}}+m_{\mathrm{a}, B r} z\left(1-\alpha_{\mathrm{SP}}\right)\right\}
\end{aligned}
$$

where $m_{\mathrm{a}, i}$, atomic mass of element $i$. Likewise,

$$
\begin{aligned}
\% N= & \frac{m_{\mathrm{a}, N} 2 x}{\left\{m_{\mathrm{a}, C}\left[19 x-24 \alpha_{\mathrm{SP}}\right]+m_{\mathrm{a}, H}\left[2 y+17 x-16 \alpha_{\mathrm{SP}}\right]+m_{\mathrm{a}, O}(2 y+3 x)\right.} \\
& \left.+m_{\mathrm{a}, N} 2 x+m_{\mathrm{a}, B} y-m_{\mathrm{a}, S i} \alpha_{\mathrm{SP}}+m_{\mathrm{a}, B r} z\left(1-\alpha_{\mathrm{SP}}\right)\right\}
\end{aligned}
$$

Solving for $x$-that is, the molar fraction of SP with respect to $\mathbf{1}$ in the final PAFgives:

$$
x=\frac{\% N \cdot 24 m_{\mathrm{a}, C} \cdot\left(2-\alpha_{\mathrm{SP}}\right)}{\% C \cdot 2 m_{\mathrm{a}, N}-\% N \cdot 19 m_{\mathrm{a}, C}}=\frac{\% N \cdot 288.24 \cdot\left(2-\alpha_{\mathrm{SP}}\right)}{\% C \cdot 28.02-\% N \cdot 228.19}
$$

which is related to the actual content of spiropyran in PAFs as

$$
\chi_{\mathrm{SP}}=\frac{x}{x+4\left(1-\alpha_{\mathrm{SP}}\right)},
$$

and finally,

$$
\chi_{\mathrm{SP}}=\frac{\% N \cdot 24 m_{\mathrm{a}, C} \cdot\left(2-\alpha_{\mathrm{SP}}\right)}{\% N \cdot 24 m_{\mathrm{a}, C} \cdot\left(2-\alpha_{\mathrm{SP}}\right)+4\left(1-\alpha_{\mathrm{SP}}\right)\left(\% C \cdot 2 m_{\mathrm{a}, N}-\% N \cdot 19 m_{\mathrm{a}, C}\right)}
$$

Solid-state NMR studies. Solid-state NMR experiments were performed on a $600-\mathrm{MHz}$ Varian VNMRS spectrometer using a triple-channel Varian fastMAS probe. Samples were packed in $1.6 \mathrm{~mm}$ rotors for magic angle sample spinning spinning rate for all experiments was $35,714 \mathrm{~Hz}$, stable to $\pm 10 \mathrm{~Hz}$. For each sample, $1 \mathrm{D}{ }^{1} \mathrm{H}$ and ${ }^{13} \mathrm{C}$ spectra were collected. All spectra were acquired at room temperature, with sample cooling by application of room temperature air at the probe head. ${ }^{1} \mathrm{H}$ spectra were acquired using a simple one-pulse sequence, where 32 or 128 scans were collected at a recycle delay of $10 \mathrm{~s} .{ }^{13} \mathrm{C}$ spectra were acquired using the SPIDAP sequence ${ }^{56} \cdot{ }^{13} \mathrm{C}$-free induction decays were recorded using the 8- $\pi$-pulse block $\{x-y-x-y-\bar{x}-\bar{y}-\bar{x}-\bar{y}\}$ repeated 20 or 32 times for an acquisition time 4.5-7 ms per transient, with a recycle delay of $4-8 \mathrm{~s}$ (ref. 56). 20, 36 and $96 \mathrm{k}$ scans were collected for the ${ }^{13} \mathrm{C}$ spectra of the small-molecule 2, PhotoPAF-3.6\% and PhotoPAN, respectively. Typical $\pi / 2$ pulse widths were 0.6 and $1.6 \mu$ s for the proton and carbon channels, respectively. Data were processed using ACD/NMR Processor 12.0 (Advanced Chemistry Development, 2007-2010, http://www.acdlabs.com). The peak at $\sim 2$ p.p.m. seen in the ${ }^{1} \mathrm{H}$ NMR spectra despite degassing of the samples can be attributed partially to residual solvent, which is corroborated by the following arguments: (i) the peak gradually decreases in spectra collected on consecutive days. For comparison, the peak at $\sim 8$ p.p.m. remained constant, (ii) ${ }^{13} \mathrm{C}$ NMR spectra from initial and final runs looked virtually the same, suggesting that the changes in the ${ }^{1} \mathrm{H}$ NMR spectra are due to the loss of residual solvent rather than sample decomposition, (iii) PAF prepared without any spiropyran $(y=0$ in Fig. 1) also shows residual peaks at $\sim 2$ p.p.m. (Supplementary Fig. 30 ), even though its structure only comprises aromatic protons, expected to appear at $\delta=8$ p.p.m., (iv) the peaks attributed to residual solvents seen in the early runs were significantly sharper than other peaks, consistent with the mobile solvent component narrowed by dynamics, which would not be possible for protons attached to the more static carbon skeleton, (v) powder samples often display the presence of solvent molecules ${ }^{15,21,22}$

\section{References}

1. Feringa, B. L. \& Browne, W. R. (eds) Molecular Switches (Wiley-VCH, 2011).

2. Klajn, R., Stoddart, J. F. \& Grzybowski, B. A. Nanoparticles functionalised with reversible molecular and supramolecular switches. Chem. Soc. Rev. 39, 2203-2237 (2010).

3. McCarthy, W. \& Powers, R. M. Thermochromic Filters and Stopband Filters for Use with Same. US Patent 20110102878 A1 filed 29 October 2010, issued 5 May (2011).

4. Burnworth, M. et al. Optically healable supramolecular polymers. Nature 472, 334-337 (2011). 
5. Ito, K. Slide-ring materials using topological supramolecular architecture. Curr. Opin. Solid State Mater. Sci 14, 28-34 (2010).

6. Ito, K., Araki, J., Suzuki, T., Yamanaka, M. \& Watanabe, K. Hydrophobic modified polyrotaxane and crosslinked polyrotaxane. US Patent 7943718 B2 filed 23 August 2006, issued 17 May (2011).

7. Coti, K. K. et al. Mechanised nanoparticles for drug delivery. Nanoscale 1, 16-39 (2009).

8. Li, Z. X., Barnes, J. C., Bosoy, A., Stoddart, J. F. \& Zink, J. I. Mesoporous silica nanoparticles in biomedical applications. Chem. Soc. Rev. 41, 2590-2605 (2012).

9. Kurihara, S. et al. Manipulation and assembly of small objects in liquid crystals by dynamical disorganizing effect of push-pull-azobenzene-dye. Sci. Rep. 3, 2167 (2013)

10. Yu, L. \& Hecht, S. Control over unfolding pathways by localizing photoisomerization events within heterosequence oligoazobenzene foldamers. Angew. Chem. Int. Ed. 52, 13740-13744 (2013).

11. van Oosten, C. L., Bastiaansen, C. W. M. \& Broer, D. J. Printed artificial cilia from liquid-crystal network actuators modularly driven by light. Nat. Mater. 8, 677-682 (2009).

12. Fischer, E. \& Hirshberg, Y. Formation of coloured forms of spirans by lowtemperature irradiation. J. Chem. Soc. 4522-4524 (1952).

13. Berkovic, G., Krongauz, V. \& Weiss, V. Spiropyrans and spirooxazines for memories and switches. Chem. Rev. 100, 1741-1753 (2000).

14. Minkin, V. I. Photo-, thermo-, solvato-, and electrochromic spiroheterocyclic compounds. Chem. Rev. 104, 2751-2776 (2004).

15. Ben, T. et al. Targeted synthesis of a porous aromatic framework with high stability and exceptionally high surface area. Angew. Chem. Int. Ed 48, 9457-9460 (2009)

16. Qiu, S. L., Zhu, G. S. \& Ben, T. Organic porous polymer materials and synthesis method thereof. European Patent 2450390 A1 filed 11 Nov. 2009, issued 9 May (2012).

17. Yuan, D. Q., Lu, W. G., Zhao, D. \& Zhou, H. C. Highly stable porous polymer networks with exceptionally high gas-uptake capacities. Adv. Mater. 23, 3723-3725 (2011).

18. Wander, M. et al. Synthesis of polyaryl rigid-core carbosilane dendrimers for supported organic synthesis. Organometallics 28, 4406-4415 (2009).

19. Fournier, J. H., Maris, T., Wuest, J. D., Guo, W. Z. \& Galoppini, E. Molecular tectonics. Use of the hydrogen bonding of boronic acids to direct supramolecular construction. J. Am. Chem. Soc. 125, 1002-1006 (2003).

20. Dattilo, D., Armelao, L., Fois, G., Mistura, G. \& Maggini, M. Wetting properties of flat and porous silicon surfaces coated with a spiropyran. Langmuir. 23, 12945-12950 (2007)

21. Stallmach, F. et al. NMR studies on the diffusion of hydrocarbons on the metalorganic framework material MOF-5. Angew. Chem. Int. Ed. 45, 2123-2126 (2006).

22. Ruiz, J. et al. Structure-solid-state CPMAS (13)C NMR correlation in palladacycle solvates (pseudo-polymorphs) with a transformation from $Z^{\prime}=1$ to $Z^{\prime}=2$. CrystEngComm 10, 1928-1938 (2008).

23. Whelan, J., Wojtyk, J. T. C. \& Buncel, E. Enhanced bistability of a photochromic microparticle in condensed medium. Chem. Mater. 20, 3797-3799 (2008).

24. Rose, M. et al. N-Heterocyclic carbene containing element organic frameworks as heterogeneous organocatalysts. Chem. Commun. 47, 4814-4816 (2011).

25. Rose, M. et al. New element organic frameworks via Suzuki coupling with high adsorption capacity for hydrophobic molecules. Soft Matter 6, 3918-3923 (2010).

26. Park, H. J. \& Suh, M. P. Mixed-ligand metal-organic frameworks with large pores: gas sorption and single-crystal-to-single-crystal transformation on guest exchange. Chem. Eur. J. 14, 8812-8821 (2008).

27. Lock, N. et al. Scrutinizing negative thermal expansion in MOF-5 by scattering techniques and ab initio calculations. Dalton. Trans. 42, 1996-2007 (2013).

28. Cabrera, I., Shvartsman, F., Veinberg, O. \& Krongauz, V. Photocontraction of liquid spiropyran-merocyanine films. Science 226, 341-343 (1984).

29. Seki, T., Ichimura, K. \& Ando, E. Stable J-aggregate formation of photoinduced merocyanine in bilayer-membrane. Langmuir 4, 1068-1069 (1988).

30. Tachibana, H., Yamanaka, Y., Sakai, H., Abe, M. \& Matsumoto, M. J-aggregate formation of amphiphilic merocyanine in Langmuir-Blodgett films. J. Lumin. 87-9, 800-802 (2000).

31. Onai, Y. et al. Colored merocyanine aggregates: long-lived crystals of large size (10-100 um) and deaggregation of small aggregates in solutions. J. Phys. Chem. 97, 9499-9505 (1993).

32. Klajn, R. Spiropyran-based dynamic materials. Chem. Soc. Rev. 43, 148-184 (2014).

33. Goldburt, E., Shvartsman, F. \& Krongauz, V. Zipper crystallization of polymers with spiropyran side groups. Macromolecules 17, 1876-1878 (1984).
34. Wismontski-Knittel, T. \& Krongauz, V. Self-assembling of spiropyran polymers by zipper crystallization. Macromolecules 18, 2124-2126 (1985).

35. Rosario, R., Gust, D., Hayes, M., Springer, J. \& Garcia, A. A. Solvatochromic study of the microenvironment of surface-bound spiropyrans. Langmuir 19, 8801-8806 (2003).

36. Kobatake, S. \& Irie, M. Single-crystalline photochromism of diarylethenes. Bull. Chem. Soc. Jpn 77, 195-210 (2004).

37. Kobatake, S., Takami, S., Muto, H., Ishikawa, T. \& Irie, M. Rapid and reversible shape changes of molecular crystals on photoirradiation. Nature 446, 778-781 (2007).

38. Koshima, H., Ojima, N. \& Uchimoto, H. Mechanical motion of azobenzene crystals upon photoirradiation. J. Am. Chem. Soc. 131, 6890-6891 (2009).

39. Benard, S. \& Yu, P. New spiropyrans showing crystalline-state photochromism. Adv. Mater. 12, 48-50 (2000).

40. Harada, J., Kawazoe, Y. \& Ogawa, K. Photochromism of spiropyrans and spirooxazines in the solid state: low temperature enhances photocoloration. Chem. Commun. 46, 2593-2595 (2010).

41. Florea, L., Scarmagnani, S., Benito-Lopez, F. \& Diamond, D. Self-assembled solvato-morphologically controlled photochromic crystals. Chem. Commun. 50, 924-926 (2014).

42. Arai, K., Shitara, Y. \& Ohyama, T. Preparation of photochromic spiropyrans linked to methyl cellulose and photoregulation of their properties. J. Mater. Chem. 6, 11-14 (1996).

43. Sakuragi, M., Aoki, K., Tamaki, T. \& Ichimura, K. The role of triplet-state of nitrospiropyran in their photochromic reaction. Bull. Chem. Soc. Jap 63, 74-79 (1990).

44. Baillet, G., Campredon, M., Guglielmetti, R., Giusti, G. \& Aubert, C. Dealkylation of N-substituted indolinospironaphthoxazine photochromic compounds under UV irradiation. J. Photochem. Photobiol. A 83, 147-151 (1994).

45. Matsushima, R., Nishiyama, M. \& Doi, M. Improvements in the fatigue resistances of photochromic compounds. J. Photochem. Photobiol. Chem. 139, 63-69 (2001)

46. Bouas-Laurent, H. \& Durr, H. Organic photochromism. Pure Appl. Chem. 73, 639-665 (2001).

47. Chen, J., Zeng, F. \& Wu, S. Z. Construction of energy transfer systems within nanosized polymer micelles and their fluorescence modulation properties. Chemphyschem 11, 1036-1043 (2010).

48. Huang, C. Q., Wang, Y., Hong, C. Y. \& Pan, C. Y. Spiropyran-based polymeric vesicles: preparation and photochromic properties. Macromol. Rapid Commun. 32, 1174-1179 (2011).

49. Ma, L. et al. Synthesis of photoresponsive spiropyran-based hybrid polymers and controllable light-triggered self-assembly study in toluene. Macromol. Chem. Phys 214, 716-725 (2013)

50. Garcia, A. A. et al. Photon-controlled phase partitioning of spiropyrans. J. Phys. Chem. A 104, 6103-6107 (2000).

51. Winkler, J. D., Deshayes, K. \& Shao, B. Photodynamic transport of metal-ions. J. Am. Chem. Soc. 111, 769-770 (1989).

52. Zhou, J. W., Li, Y. T. \& Song, X. Q. Investigation of the chelation of a photochromic spiropyran with $\mathrm{Cu}(\mathrm{II})$. J. Photochem. Photobiol. A 87, 37-42 (1995).

53. Ferraudi, G. \& Muralidharan, S. Photochemical properties of coppercomplexes. Coord. Chem. Rev. 36, 45-88 (1981).

54. Cervone, E., Camassei, F. D., Giannini, I. \& Sykora, J. Photoredox behavior of chlorocopper (II) complexes in acetonitrile - mechanism and quantum yields. J. Photochem 11, 321-332 (1979).

55. Neilson, B. M. \& Bielawski, C. W. Illuminating photoswitchable catalysis. ACS Catalysis 3, 1874-1885 (2013).

56. Lupulescu, A. \& Frydman, L. Sensitizing solid state nuclear magnetic resonance of dilute nuclei by spin-diffusion assisted polarization transfers. J. Chem. Phys 135, 134202 (2011)

\section{Acknowledgements}

This work was supported by the Israel Science Foundation (grant no. 1463/11) and by the Minerva Foundation with funding from the Federal German Ministry for Education and Research. We gratefully acknowledge Dr Christophe Coudret (Université Paul Sabatier) for helpful discussions and Ms Aleksandra Geller for critical reading of the manuscript. We thank Mr Yotam Assher for his assistance with TGA and infrared spectroscopy analysis, Drs Ronit Popovitz-Biro and Pradipta Sankar Maiti for carrying out highresolution TEM studies, Dr Gregory Leitus for solving the crystal structure of 1, Professor Lucio Frydman for the access to his NMR facility, and Dr Vlad Brumfeld for initial fluorescence microscopy studies.

\section{Author contributions}

P.K.K. synthesized all new compounds and materials, performed photochromism, acidochromism and ionochromism studies. G.O. performed solid-state NMR studies 
V.K. performed fluorescence microscopy studies. R.K. conceived the project, supervised the experiments and wrote the manuscript.

\section{Additional information}

Accession codes: The X-ray crystallographic coordinates for structures reported in this article have been deposited at the Cambridge Crystallographic Data Centre (CCDC), under deposition number CCDC 978998. These data can be obtained free of charge from The Cambridge Crystallographic Data Centre via http://www.ccdc.cam.ac.uk/data_ request/cif.

Supplementary Information accompanies this paper at http://www.nature.com/nature communications

Competing financial interests: The authors declare no competing financial interests.
Reprints and permission information is available online at http://npg.nature.com/ reprintsandpermissions

How to cite this article: Kundu, P. K. et al. Nanoporous frameworks exhibiting multiple stimuli responsiveness. Nat. Commun. 5:3588 doi: 10.1038/ncomms4588 (2014).

(c) (i) $\odot$ This work is licensed under a Creative Commons Attribution(c) ${ }_{\mathrm{BY}}$ NC ND NonCommercial-NoDerivs 3.0 Unported License. The images or other third party material in this article are included in the article's Creative Commons license, unless indicated otherwise in the credit line; if the material is not included under the Creative Commons license, users will need to obtain permission from the license holder to reproduce the material. To view a copy of this license, visit http://creativecommons. org/licenses/by-nc-nd/3.0/ 